\title{
REVERSE HÖLDER INEQUALITIES FOR SPHERICAL HARMONICS
}

\author{
JAVIER DUOANDIKOETXEA
}

(Communicated by Richard R.Goldberg)

\begin{abstract}
We prove that the $L^{p}$-norm with respect to the normalized Lebesgue measure on the sphere of any spherical harmonic of degree $k$ is bounded by a constant independent of the dimension times its $L^{2}$-norm. Several consequences are obtained from this result.
\end{abstract}

1. Introduction. In a previous paper [2] we were led to study the quotient $\left\|Y_{k}\right\|_{p} /\left\|Y_{k}\right\|_{2}$ where $Y_{k}$ stands for a spherical harmonic of degree $k$ in $\mathbf{R}^{n}$ and the $L^{p}$-norms are taken with respect to the normalized Lebesgue measure on the sphere $S^{n-1}$, i.e.,

$$
\left\|Y_{k}\right\|_{p}^{p}=\frac{1}{\left|S^{n-1}\right|} \int_{S^{n-1}}\left|Y_{k}(u)\right|^{p} d \sigma(u)
$$

$\left(d \sigma(u)=\right.$ Lebesgue measure on $S^{n-1},\left|S^{n-1}\right|=$ measure of $\left.S^{n-1}=2 \pi^{n / 2} \Gamma(n / 2)^{-1}\right)$.

When $p>2$, Hölder's inequality provides the trivial lower bound 1 . We prove here, by using two different approaches, that we have the upper bound $(p-1)^{k / 2}$, independent of $n$. The first proof is the same as in [2] with further precision; the second uses two well-known but far from trivial facts: the Bochner-Hecke formula and the Beckner-Hausdorff-Young inequality.

For $p<2$, Hölder's inequality gives the upper bound 1 and a lower bound independent of $n$ can be found by using the preceding part and interpolation.

Similar results can be obtained for any polynomial of degree $k$ and any sphere in $\mathbf{R}^{n}$ using the decomposition of the polynomial restricted to the sphere as a sum of spherical harmonics. Some other consequences are also given.

We use the same notation for the spherical harmonic $Y_{k}$ defined on $S^{n-1}$ and the solid harmonic defined in all $\mathbf{R}^{n}$ which are related by $Y_{k}(x)=Y_{k}(x /|x|)|x|^{k}$.

We are indebted to José L. Rubio de Francia for suggesting certain applications and improvements to this paper.

\section{The main theorem and its two proofs.}

THEOREM 1. Let $Y_{k}$ be a spherical harmonic of degree $k$ in $\mathbf{R}^{n}$. Then, if $p \geq 2$,

$$
\left\|Y_{k}\right\|_{p} \leq(p-1)^{k / 2}\left\|Y_{k}\right\|_{2} \text {. }
$$

PROOF. We use induction on $k$. Let $k=1$. By rotation it is enough to prove the theorem for $Y_{1}(u)=u_{1}$. But a simple computation gives

$$
\frac{1}{\left|S^{n-1}\right|} \int_{S^{n-1}}\left|u_{1}\right|^{p} d \sigma(u)=\frac{\Gamma(n / 2) \Gamma((p+1) / 2)}{\pi^{1 / 2} \Gamma((n+p) / 2)}
$$

Received by the editors July 14, 1986 .

1980 Mathematics Subject Classification (1985 Revision). Primary 42C05. 
and $\left\|Y_{1}\right\|_{2}=n^{-1 / 2}$; then it follows from the properties of the gamma function that

$$
\left\|Y_{1}\right\|_{p} /\left\|Y_{1}\right\|_{2} \leq(p-1)^{1 / 2} \text {. }
$$

Assume now the theorem for $k-1$ and let $Y_{k}$ be a spherical harmonic of degree $k$. We claim that it is enough to obtain

$$
\left\|Y_{k}\right\|_{p} \leq\left(\frac{p-1}{k(k p+n-2)}\right)^{1 / 2}\left\|\left|\nabla Y_{k}\right|\right\|_{p} \quad \text { with equality for } p=2 \text {. }
$$

In fact, the induction hypothesis and Minkowski's inequality imply

$$
\left\|\left|\nabla Y_{k}\right|\right\|_{p} \leq(p-1)^{(k-1) / 2}\left\|\left|\nabla Y_{k}\right|\right\|_{2}
$$

and then using (1) successively with $L^{p}$ - and $L^{2}$-norms, we get

$$
\begin{aligned}
\left\|Y_{k}\right\|_{p} & \leq\left(\frac{p-1}{k(k p+n-2)}\right)^{1 / 2}(p-1)^{(k-1) / 2}\left\|\left|\nabla Y_{k}\right|\right\|_{2} \\
& =(p-1)^{k / 2}\left(\frac{2 k+n-2}{k p+n-2}\right)^{1 / 2}\left\|Y_{k}\right\|_{2} \leq(p-1)^{k / 2}\left\|Y_{k}\right\|_{2} .
\end{aligned}
$$

Finally we prove (1). From the homogeneity of solid harmonic $Y_{k}$ and Green's formula we have

$$
\begin{aligned}
\int_{S^{n-1}}\left|Y_{k}\right|^{p} d \sigma & =\frac{1}{k} \int_{S^{n-1}}\left|Y_{k}\right|^{p-1} \operatorname{sgn} Y_{k} \frac{\partial Y_{k}}{\partial \nu} d \sigma \\
& =\frac{1}{k} \int_{|x|<1} \nabla\left(\left|Y_{k}\right|^{p-1} \operatorname{sgn} Y_{k}\right) \nabla Y_{k} d x \\
& =\frac{p-1}{k} \int_{|x|<1}\left|Y_{k}\right|^{p-2}\left|\nabla Y_{k}\right|^{2} d x \\
& =\frac{p-1}{k(k p+n-2)} \int_{S^{n-1}}\left|Y_{k}\right|^{p-2}\left|\nabla Y_{k}\right|^{2} d \sigma
\end{aligned}
$$

When $p=2$ we get the equality in (1); for $p>2$, just apply Hölder's inequality with exponents $p /(p-2)$ and $p / 2$.

The second way to prove Theorem 1 gives a somewhat more precise result:

THEOREM $1^{\prime}$. Let $Y_{k}$ be a spherical harmonic of degree $k, 2 \leq p<\infty$ and $1 / p+1 / q=1$. Then,

$$
\left\|Y_{k}\right\|_{p} \leq(p-1)^{k / 2}\left\|Y_{k}\right\|_{q} .
$$

Theorem 1 follows immediately because of $\left\|Y_{k}\right\|_{q} \leq\left\|Y_{k}\right\|_{2}$.

PROOF. Bochner-Hecke's formula states that

$$
\mathfrak{F}\left(e^{-\pi|x|^{2}} Y_{k}(x)\right)=i^{-k} e^{-\pi|\xi|^{2}} Y_{k}(\xi)
$$

( $\mathfrak{F}$ is the Fourier transform and $Y_{k}$ is here the solid harmonic; see Stein [4, p. 71].) The Hausdorff-Young inequality can be written in the form

$$
\|\mathfrak{F}(f)\|_{L^{p}\left(\mathbf{R}^{n}\right)} \leq\left(q^{1 / q} / p^{1 / p}\right)^{n / 2}\|f\|_{L^{q}\left(\mathbf{R}^{n}\right)}
$$

(see Beckner [1]). When this inequality is applied to (2), taking into account that

$$
\left\|e^{-\pi|x|^{2}} Y_{k}(x)\right\|_{L^{p}\left(\mathbf{R}^{n}\right)}^{p}=\frac{\Gamma((k p+n) / 2)}{\pi^{k p / 2} p^{(k p+n) / 2} \Gamma(n / 2)}\left\|Y_{k}\right\|_{p}^{p}
$$


we get

$$
\frac{\left\|Y_{k}\right\|_{p}}{\left\|Y_{k}\right\|_{q}} \leq\left(\frac{p}{q}\right)^{k / 2} \frac{\Gamma((k q+n) / 2)^{1 / q} \Gamma(n / 2)^{1 / p}}{\Gamma((k p+n) / 2)^{1 / p} \Gamma(n / 2)^{1 / q}} .
$$

Since $\log \Gamma$ is a convex function in $(0, \infty)$, the last factor is $\leq 1$ and the theorem is proved.

The function $Y_{k}(x)=\left(x_{1}+i x_{2}\right)^{k}$ is harmonic and homogeneous of degree $k$. If we compute the $L^{p}$-norm of its restriction to $S^{n-1}$ we see that the preceding results are sharp in the following sense: for constants independent of $n$, no exponent less than $k / 2$ can appear in the right-hand side of Theorems 1 and $1^{\prime}$; in fact

$$
\sup _{n} \frac{\left\|Y_{k}\right\|_{p}}{\left\|Y_{k}\right\|_{2}} \leq c(k) p^{k / 2} \text {. }
$$

3. Some consequences. (a) Theorem 1 and the method of rotations give the following: Let $\left\{Y_{j}\right\}$ be a basis of the linear space of spherical harmonics of degree $k$ in $\mathbf{R}^{n}$ and $d(k, n)$ its dimension. If we normalize the $Y_{j}$ in such a way that $\left\|Y_{j}\right\|_{2}=d(k, n)^{-1 / 2}$ and define the operators $\left(R_{j} f\right)^{-}(\xi)=Y_{j}(\xi /|\xi|) \hat{f}(\xi)$, there then exists $C_{p, k}$ independent of $n$ such that

$$
\left\|\left(\sum_{j=1}^{d(k, n)}\left|R_{j} f\right|^{2}\right)^{1 / 2}\right\|_{p} \leq C_{p, k}\|f\|_{p}, \quad 1<p<\infty
$$

with $C_{p, k}=O\left(p^{1+k / 2}\right), p \rightarrow \infty$, and $=O\left((p-1)^{-1}\right), p \rightarrow 1$. This was our motivation for Theorem 1 and can be seen in [2].

(b) For $p<2$, a lower bound for $\left\|Y_{k}\right\|_{p} /\left\|Y_{k}\right\|_{p}$ can be obtained from Theorem 1, namely

COROLlaRY 2. If $0<p<2$,

$$
\left\|Y_{k}\right\|_{2} \leq e^{k((2 / p)-1)}\left\|Y_{k}\right\|_{p}
$$

PROOF. Let $s>2$. By interpolation and Theorem 1

$$
\left\|Y_{k}\right\|_{2} \leq\left\|Y_{k}\right\|_{p}^{\theta}\left\|Y_{k}\right\|_{s}^{1-\theta} \leq(s-1)^{(1-\theta) k / 2}\left\|Y_{k}\right\|_{p}^{\theta}\left\|Y_{k}\right\|_{2}^{1-\theta}
$$

with $1 / 2=\theta / p+(1-\theta) / s$. Then,

$$
\left\|Y_{k}\right\|_{2} \leq(s-1)^{(1-\theta) / \theta \cdot k / 2}\left\|Y_{k}\right\|_{p}
$$

and the corollary follows from

$$
\inf _{s>2}(s-1)^{(1-\theta) / \theta}=\lim _{s \rightarrow 2}(s-1)^{(1-\theta) / \theta}=e^{2(2 / p-1)} .
$$

(c) Theorem 1 and Corollary 2 have the following similar versions in the case of arbitrary polynomials.

COROLlaRY 3. Let $P_{k}$ be any polynomial of degree $k$ and $S$ any sphere in $\mathbf{R}^{n}$. Then, if $2<p<\infty$,

$$
\left(\frac{1}{|S|} \int_{S}\left|P_{k}\right|^{p} d \sigma\right)^{1 / p} \leq p^{k / 2}\left(\frac{1}{|S|} \int_{S}\left|P_{k}\right|^{2} d \sigma\right)^{1 / 2}
$$


and if $0<p<2$,

$$
\left(\frac{1}{|S|} \int_{S}\left|P_{k}\right|^{2} d \sigma\right)^{1 / 2} \leq 4^{k(2 / p-1)}\left(\frac{1}{|S|} \int_{S}\left|P_{k}\right|^{p} d \sigma\right)^{1 / p} .
$$

PROOF. Since translation and dilation change a polynomial into another of the same degree, it will be enough to prove the result for $S=S^{n-1}$. But on $S^{n-1}$, $P_{k}=\sum_{j=0}^{k} Y_{j}$ where $Y_{j}$ is a spherical harmonic of degree $j$. By Theorem 1 ,

$$
\left\|P_{k}\right\|_{p} \leq \sum_{j=0}^{k}\left\|Y_{j}\right\|_{p} \leq \sum_{j=0}^{k}(p-1)^{j / 2}\left\|Y_{j}\right\|_{2}
$$

and the first part of the corollary is a consequence of the orthogonality of the $Y_{j}$ after applying Cauchy-Schwarz inequality.

The second part follows from the first as in the proof of Corollary 2 .

(d) The size of the constants in Theorem 1 and Corollary 3 makes it possible to give an estimate of exponential type with constant independent of $n$.

COROLlaRY 4. Let $P_{k}$ be a polynomial of degree $k$ in $\mathbf{R}^{n}$. Then, for any sphere $S$ in $\mathbf{R}^{n}$

$$
\frac{1}{|S|} \int_{S} \exp \left|\frac{P_{k}(u)}{\left\|P_{k}\right\|_{2}}\right|^{\lambda} d \sigma(u) \leq C(k, \lambda)
$$

with a constant independent of $n$ if $\lambda<2 / k$ (also for $\lambda=2 / k$ if $k \geq 6$ ).

(e) The following application is based on a result in probability theory.

Let $Y^{(n)}=\left(Y_{1}, \ldots, Y_{n}, 0,0, \ldots\right), n=1,2, \ldots$, be random variables such that $\left(Y_{1}, \ldots, Y_{n}\right)$ are uniformly distributed in a sphere of $\mathbf{R}^{n}$ of radius $r_{n}=(n / 2 \pi)^{1 / 2}$ and let $X=\left(X_{1}, \ldots, X_{m}, \ldots\right)$ be a random variable with $X_{1}, \ldots, X_{m}, \ldots$ independent and having $\exp \left(-\pi|x|^{2}\right)$ as distribution function. Then, the sequence $Y^{(n)}$ converges to $X$ in law.

This result is known and can be easily proved. If $E_{n}(f)$ stands for the expectation of $f$ with respect to $Y^{(n)}$ and $E(f)$ is the expectation with respect to $X$, we have

$$
\lim _{n \rightarrow \infty} E_{n}(f)=E(f) \text {. }
$$

Taking now as $f$ the $p$ th power of a polynomial of degree $k$ and using Corollary 3 we have

CoROllaRY 5. Let $X=\left(X_{1}, \ldots, X_{n}, \ldots\right)$ be a random variable where the $X_{i}$ are independent and have $\exp \left(-\pi|x|^{2}\right)$ as distribution function. If $P(X)$ is a polynomial of degree $k$ in the variables $X_{1}, \ldots, X_{n}, \ldots$ the following reverse Hölder inequalities hold.

$$
\begin{aligned}
& E\left(|P|^{p}\right)^{1 / p} \leq p^{k / 2} E\left(|P|^{2}\right)^{1 / 2}, \quad 2<p<\infty \\
& E\left(|P|^{2}\right)^{1 / 2} \leq 4^{k(2 / p-1)} E\left(|P|^{p}\right)^{1 / p}, \quad 0<p<2 .
\end{aligned}
$$

(f) Upper bounds for the quotient $\left\|Y_{k}\right\|_{p} /\left\|Y_{k}\right\|_{2}, 2<p<\infty$, are interesting also in the study of Bochner-Riesz operators on the sphere. In that case the dimension $n$ of the underlying space is kept fixed and the interest is in the behaviour of the quotient for $k \rightarrow \infty$. Sharp bounds have been obtained by C. Sogge [3].

$$
\left\|Y_{k}\right\|_{p} /\left\|Y_{k}\right\|_{2}=O\left(k^{\alpha(p)}\right)
$$


where

$$
\begin{aligned}
\alpha(p) & =(n-2)(p-2) / 4 p \quad \text { if } 2 \leq p \leq 2 n /(n-2), \\
& =(n-2) / 2-(n-1) / p \quad \text { if } 2 n /(n-2) \leq p \leq \infty .
\end{aligned}
$$

From the proof of Theorem $1^{\prime}$ and more precisely from inequality (3) it follows immediately that

$$
\left\|Y_{k}\right\|_{p} /\left\|Y_{k}\right\|_{q}=O\left(k^{(n-1)(p-2) / 2 p}\right) .
$$

But, if we put $\left\|Y_{k}\right\|_{2}$ instead of $\left\|Y_{k}\right\|_{q}$ the bound we get for the quotient is not sharp and it does not apply to obtaining nontrivial results for Bochner-Riesz operators.

Using (4) we can get in a trivial way the bound $O\left(k^{2 \alpha(p)}\right)$ for the quotient $\left\|Y_{k}\right\|_{p} /\left\|Y_{k}\right\|_{q}$. It is easily verified that $2 \alpha(p)$ is less than our exponent when $p$ is close to 2 and bigger when $p$ is close to $\infty$ (for $n>3$ ).

\section{REFERENCES}

1. W. Beckner, Inequalities in Fourier analysis, Ann. of Math. (2) 102 (1975), 159-182.

2. J. Duoandikoetxea and J. L. Rubio de Francia, Estimations indépendantes de la dimension pour les transformées de Riesz, C.R. Acad. Sci. Paris 300 (1985), 193-196.

3. C. Sogge, Oscillatory integrals and spherical harmonics, Duke Math. J. 53 (1986), 43-65.

4. E. M. Stein, Singular integrals and differentiability properties of functions, Princeton Univ. Press, Princeton, N.J., 1970.

División de MAtemáticas, Universidad Autónoma, 28049 Madrid, SPAin 